\title{
A New Fourth Order Difference Approximation for the Solution of Three-Dimensional Non-Linear Biharmonic Equations Using Coupled Approach
}

\author{
Ranjan Kumar Mohanty ${ }^{1}$, Mahinder Kumar Jain ${ }^{2 *}$, Biranchi Narayan Mishra ${ }^{3 \#}$ \\ ${ }^{1}$ Department of Mathematics, Faculty of Mathematical Sciences, University of Delhi, Delhi, India \\ ${ }^{2}$ Department of Mathematics, Indian Institute of Technology, New Delhi, India \\ ${ }^{3}$ Department of Mathematics, Utkal University, Bhubaneswar, India \\ E-mail: rmohanty@maths.du.ac.in,bn_misramath@hotmail.com \\ Received October 13, 2011; revised November 2, 2011; accepted November 9, 2011
}

\begin{abstract}
This paper deals with a new higher order compact difference scheme, which is, $O\left(h^{4}\right)$ using coupled approach on the 19-point 3D stencil for the solution of three dimensional nonlinear biharmonic equations. At each internal grid point, the solution $u(x, y, z)$ and its Laplacian $\nabla^{2} u$ are obtained. The resulting stencil algorithm is presented and hence this new algorithm can be easily incorporated to solve many problems. The present discretization allows us to use the Dirichlet boundary conditions only and there is no need to discretize the derivative boundary conditions near the boundary. We also show that special treatment is required to handle the boundary conditions. Convergence analysis for a model problem is briefly discussed. The method is tested on three problems and compares very favourably with the corresponding second order approximation which we also discuss using coupled approach.
\end{abstract}

Keywords: Three-Dimensional Non-Linear Biharmonic Equation, Finite Differences, Fourth Order Accuracy, Compact Discretization, Block-Block-Tridiagonal, Tangential Derivatives, Laplacian, Stream Function, Reynolds Number

\section{Introduction}

We are interested to develop a new algorithm for solving the three-dimensional non-linear biharmonic partial differential equation

$$
\begin{aligned}
& \nabla^{4} u(x, y, z) \equiv \frac{\partial^{4} u}{\partial x^{4}}+\frac{\partial^{4} u}{\partial y^{4}}+\frac{\partial^{4} u}{\partial z^{4}} \\
& +2\left(\frac{\partial^{4} u}{\partial x^{2} \partial y^{2}}+\frac{\partial^{4} u}{\partial y^{2} \partial z^{2}}+\frac{\partial^{4} u}{\partial z^{2} \partial x^{2}}\right) \\
& =f\left(x, y, z, u, u_{x}, u_{y}, u_{z}, \nabla^{2} u, \nabla^{2} u_{x}, \nabla^{2} u_{y}, \nabla^{2} u_{z}\right) \\
& 0<x, y, x<1
\end{aligned}
$$

defined in the solution region $\Omega=\{(x, y, z) \mid 0<x, y, z<1\}$ with boundary $\partial \Omega$, where $\nabla^{2} u(x, y, z) \equiv \frac{\partial^{2} u}{\partial x^{2}}+\frac{\partial^{2} u}{\partial y^{2}}+\frac{\partial^{2} u}{\partial z^{2}}$ represents the three di-

"Present address: 4076, C/4, Vasant Kunj, New Delhi, India.

"Present address: Department of Mathematics, Rajasunakhala College, Nayagarh, Orissa, India. mensional Laplacians of $u$ in $\Omega$. We assume that the solution $u(x, y, z)$ is smooth enough to maintain the order and accuracy of the algorithm as high as possible under consideration.

The Dirichlet boundary conditions are given by

$$
u=f_{1}(x, y, z), \frac{\partial^{2} u}{\partial n^{2}}=f_{2}(x, y, z),(x, y, z) \in \partial \Omega .
$$

The nonlinear biharmonic equation is a fourth order elliptic partial differential equation which occurs in many areas of physics and applied mathematics, especially in elasticity theory and stokes flow problems. It occurs with fluid flowing over an obstacle or with the movement of a natural or artificial body. Common examples are the flows past an airplane, a submarine, an automobile, or wind blowing past a high-rise building. Till about three and half decades ago, second order accuracy was considered to be sufficient for most biharmonic problems. In particular, the central difference schemes have been the most popular ones because of their straightforwardness 
in application. Though for problems having well behaved solutions, the solution may be of poor quality for convection dominated flows, or for high Reynolds number if the mesh is not sufficiently refined. Again, higher order discretization is generally associated with non-compact stencil which increase the band-width of the resultant coefficient matrix. Both mesh refinement and increased matrix band-width invariably lead to a large number of arithmetic operations. Thus neither a lower order accurate method on a fine mesh nor a higher order accurate one on a non-compact stencil seems to be computationally cost-effective. This is where higher order compact (HOC) finite difference methods become important. A compact finite difference schemes is one which utilizes grid points located only directly adjacent to the node about which differences are taken. In addition, if the scheme has an accuracy greater than two, it is termed as HOC method. The higher order accuracy of the HOC methods combined with the compactness of the difference stencils yields highly accurate numerical solutions on relatively coarser grids with greater computational efficiency. A compact difference scheme is one that is restricted to the patch of cells immediately surrounding any given node and does not extend further. Most standard difference schemes such as the central difference scheme for second order elliptic partial differential equations are compact. The high order compact method considered here is different in that the governing differential equation is used to approximate the lower order derivative terms with the imbedding technique. The scheme is difficult to develop due to the need for extensive algebraic manipulation, especially for non-linear problems. However, once high-order method developed, it can be incorporated easily in application. Various approaches for the numerical solution of 2D biharmonic problems has been discussed in the literature. Not many authors have tried to solve the three-dimensional biharmonic problems. The reason is that for high order approximation it is difficult to discretize the nonlinear biharmonic equation as well as the associated boundary conditions using a single computational cell. Another reason of necessity is that three-dimensional problems require large computing power and place huge amount of memory requirements on the computational systems. Such computing power has only recently begun to become available for academic research. Smith [1] and Ehrlich [2,3] have used coupled approach and solved 2D biharmonic equation using second order accurate finite difference equations. Bauer and Riess [4] have used block iterative method to solve block 5-diagonal matrices for the solution of 2D biharmonic problem of first kind. Glowinski and Pironneau [5] have developed a stable lower order numerical method for the first biharmonic problem. Later, kwon et al. [6], Stephenson [7], and Mohanty et al. [8-12] have developed certain second- and fourth-order finite difference methods for the solution of two and three dimensional biharmonic problems using single compact cells. Recently, using more grid points Singh et al [13] and Khattar et al. [14] have discussed the fourth order numerical methods for the solution of 2D and 3D biharmonic problems of second kind. Further, using coupled approach Mohanty [15] has derived a new nine point fourth order finite difference method for the solution of non-linear biharmonic problems of second kind. In most recently, using nine point compact cell Mohanty et al. [16] have discussed fourth order finite difference method for the solution of nonlinear 2D triharmonic problems. Earlier, Mohanty et al. [17-19] have developed fourth order compact difference schemes for the solution of multidimensional non-linear elliptic partial differential equations. Fourth order compact difference schemes have become quite popular as against the other lower order accurate schemes which require high mesh refinement and hence are computationally inefficient. On the other hand, the higher order accuracy of the fourth order compact methods combined with the compactness of the difference stencil yields highly accurate numerical solutions on relatively coarser grids with greater computational efficiency. A conventional approach for solving the 3D biharmonic equation is to discretize the differential Equation (1) on a uniform grid using 125-point approximations with truncation error of order $h^{2}$. This approximation connect the values of central point in terms of 124 neighbouring values of $u$ in $5 \times 5 \times 5$ cubic grid. We note that the central value of $u$ is connected to grid points two grids away in each direction from the central point and the difference approximations needs to be modified at grid points near the boundaries. There are serious computational difficulties with solution of the linear and non-linear systems obtained through 125-point discretization of the $3 \mathrm{D}$ biharmonic equation. Approximations using compact cells avoid these difficulties. The compact approach involves discretizing the biharmonic equations using not just the grid values of the unknown solution $u$ but also the values of the derivatives $u_{x x}, u_{y y}$ and $u_{z z}$ at selected grid points (see Mohanty and Pandey [10]). It is required to solve the system of four equations to obtain the solutions of $u, u_{x x}, u_{y y}$ and $u_{z z}$. In this paper, we write the original differential equation in a coupled manner and introduce new concepts to handle the boundary conditions without discretizing them. For fourth order approximations we use only 19-point compact cell (see Figure 1). No approximations for derivatives need to be carried out at the boundaries and the given Dirichlet boundary conditions are exactly satisfied. The main advantage of this work is that we require to solve a system of two equations, whereas in our previous work [10], we were required to solve a system of four equations to ob- 


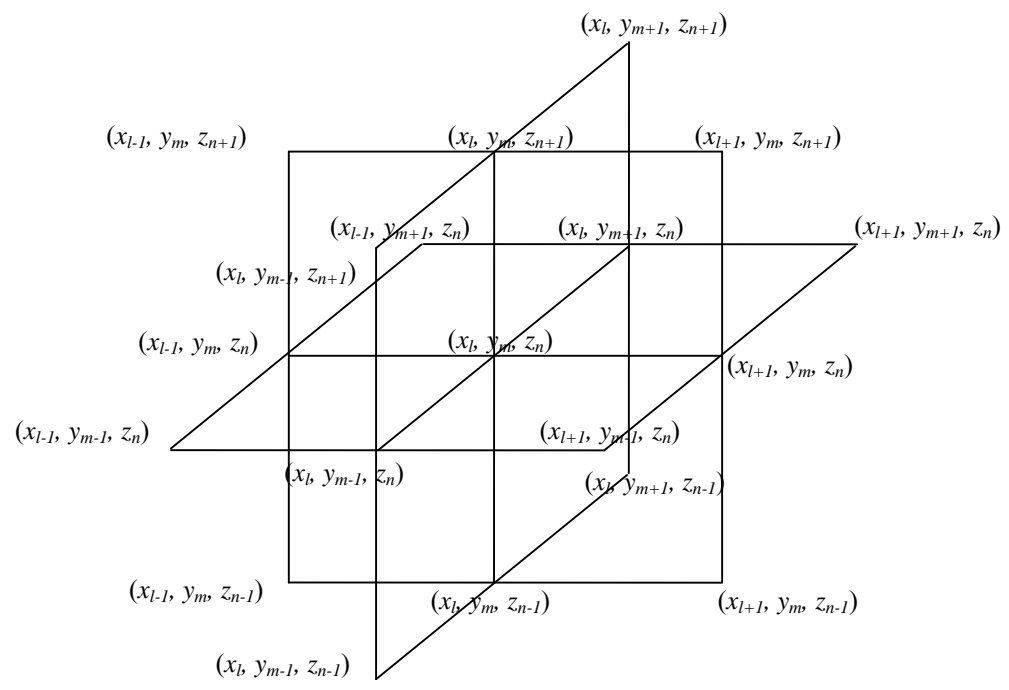

Figure 1. 19-point 3D single computational cell.

tain the numerical solution of $u(x, y, z)$. Thus the proposed method requires less algebraic operations as compared to the method discussed in [10]. In Section 2, we give the formulation of the method. The complete derivation of the method is carried out in Section 3. In order to validate the proposed fourth order method and test its robustness, we solve three problems in Section 4 and also discussed stability analysis briefly for a model problem. Concluding remarks are presented in Section 5.

\section{Formulation of the Fourth Order Discretization}

Consider the three-dimensional solution region $\Omega$, which is replaced by a set of grid points $\left(x_{l}, y_{m}, z_{n}\right)$, where $h>0$ is the mesh sizes in $x$-, $y$ - and $z$ - directions, and grid points are defined by $x_{l}=l h, y_{m}=m h, z_{n}=n h ; l$, $m, n=0(1) N+1$ with $(N+1) h=1$. Let $u_{l, m, n}$ and $U_{l, m, n}$ be the approximate and exact solution values of $u(x, y, z)$ at the grid point $\left(x_{l}, y_{m}, z_{n}\right)$, respectively.

The Dirichlet boundary conditions are given by (2). Since the grid lines are parallel to coordinate axes and the values of $u$ are exactly known on the boundary, this implies, the successive tangential partial derivatives of $u$ are known exactly on the boundary. For example, on the plane $y=0$, the values of $u(x, 0, z)$ and $u_{y y}(x, 0, z)$ are known, i.e., the values of $u_{x}(x, 0, z), u_{z}(x, 0, z)$, $u_{x x}(x, 0, z), u_{z z}(x, 0, z), \cdots$ etc are known on the plane $y=0$. This implies, the values of $u(x, 0, z)$ and $\nabla^{2} u(x, 0, z) \equiv u_{x x}(x, 0, z)+u_{y y}(x, 0, z)+u_{z z}(x, 0, z) \quad$ are known on the plane $y=0$. Similarly the values of $u$ and $\nabla^{2} u$ are known on all plane sides of the cubic region $\Omega$.

Let us denote $\nabla^{2} u(x, y, z)=v(x, y, z)$. Then we re- formulate the boundary value problems (1) and (2) in a coupled manner as

$$
\begin{gathered}
\nabla^{2} u(x, y, z) \equiv \frac{\partial^{2} u}{\partial x^{2}}+\frac{\partial^{2} u}{\partial y^{2}}+\frac{\partial^{2} u}{\partial z^{2}}=v(x, y, z), \\
(x, y, z) \in \Omega \\
\nabla^{2} v(x, y, z) \equiv \frac{\partial^{2} v}{\partial x^{2}}+\frac{\partial^{2} v}{\partial y^{2}}+\frac{\partial^{2} v}{\partial z^{2}} \\
=f\left(x, y, z, u, v, u_{x}, v_{x}, u_{y}, v_{y}, u_{z}, v_{z}\right), . \\
(x, y, z) \in \Omega
\end{gathered}
$$

subject to the Dirichlet boundary conditions prescribed by

$$
u=a(x, y, z), \quad v=c(x, y, z), \quad(x, y, z) \in \partial \Omega .
$$

Let at the grid points $\left(x_{l}, y_{m}, z_{n}\right)$, the approximate and exact solution values of $v(x, y, z)$ be denoted as $v_{l, m, n}$ and $V_{l, m, n}$, respectively.

In order to obtain fourth order approximations on the 19-point compact cell for the system of non-linear differential Equations (3a) and (3b), we need the following approximations:

$$
\begin{aligned}
& \bar{U}_{x l, m, n}=\frac{1}{2 h}\left(U_{l+1, m, n}-U_{l-1, m, n}\right), \\
& \bar{V}_{x l, m, n}=\frac{1}{2 h}\left(V_{l+1, m, n}-V_{l-1, m, n}\right), \\
& \bar{U}_{y l, m, n}=\frac{1}{2 h}\left(U_{l, m+1, n}-U_{l, m-1, n}\right), \\
& \bar{V}_{y l, m, n}=\frac{1}{2 h}\left(V_{l, m+1, n}-V_{l, m-1, n}\right), \\
& \bar{U}_{z l, m, n}=\frac{1}{2 h}\left(U_{l, m, n+1}-U_{l, m, n-1}\right),
\end{aligned}
$$




$$
\begin{aligned}
& \bar{V}_{z l, m, n}=\frac{1}{2 h}\left(V_{l, m, n+1}-V_{l, m, n-1}\right), \\
& \bar{U}_{x l \pm 1, m, n}=\frac{1}{2 h}\left( \pm 3 U_{l \pm 1, m, n} \mp 4 U_{l, m, n} \pm U_{l \mp 1, m, n}\right), \\
& \bar{V}_{x l \pm 1, m, n}=\frac{1}{2 h}\left( \pm 3 V_{l \pm 1, m, n} \mp 4 V_{l, m, n} \pm V_{l \mp 1, m, n}\right), \\
& \bar{U}_{x l, m \pm 1, n}=\frac{1}{2 h}\left(U_{l+1, m \pm 1, n}-U_{l-1, m \pm 1, n}\right), \\
& \bar{V}_{x l, m \pm 1, n}=\frac{1}{2 h}\left(V_{l+1, m \pm 1, n}-V_{l-1, m \pm 1, n}\right) \text {, } \\
& \bar{U}_{x l, m, n \pm 1}=\frac{1}{2 h}\left(U_{l+1, m, n \pm 1}-U_{l-1, m, n \pm 1}\right), \\
& \bar{V}_{x l, m, n \pm 1}=\frac{1}{2 h}\left(V_{l+1, m, n \pm 1}-V_{l-1, m, n \pm 1}\right) \text {, } \\
& \bar{U}_{y l \pm 1, m, n}=\frac{1}{2 h}\left(U_{l \pm 1, m+1, n}-U_{l \pm 1, m-1, n}\right), \\
& \bar{V}_{y l \pm 1, m, n}=\frac{1}{2 h}\left(V_{l \pm 1, m+1, n}-V_{l \pm 1, m-1, n}\right), \\
& \bar{U}_{y l, m \pm 1, n}=\frac{1}{2 h}\left( \pm 3 U_{l, m \pm 1, n} \mp 4 U_{l, m, n} \pm U_{l, m \mp 1, n}\right), \\
& \bar{V}_{y l, m \pm 1, n}=\frac{1}{2 h}\left( \pm 3 V_{l, m \pm 1, n} \mp 4 V_{l, m, n} \pm V_{l, m \mp 1, n}\right) \text {, } \\
& \bar{U}_{y l, m, n \pm 1}=\frac{1}{2 h}\left(U_{l, m+1, n \pm 1}-U_{l, m-1, n \pm 1}\right), \\
& \bar{V}_{y l, m, n \pm 1}=\frac{1}{2 h}\left(V_{l, m+1, n \pm 1}-V_{l, m-1, n \pm 1}\right) \text {, } \\
& \bar{U}_{z l \pm 1, m, n}=\frac{1}{2 h}\left(U_{l \pm 1, m, n+1}-U_{l \pm 1, m, n-1}\right), \\
& \bar{V}_{z l \pm 1, m, n}=\frac{1}{2 h}\left(V_{l \pm 1, m, n+1}-V_{l \pm 1, m, n-1}\right) \text {, } \\
& \bar{U}_{z l, m \pm 1, n}=\frac{1}{2 h}\left(U_{l, m \pm 1, n+1}-U_{l, m \pm 1, n-1}\right), \\
& \bar{V}_{z l, m \pm 1, n}=\frac{1}{2 h}\left(V_{l, m \pm 1, n+1}-V_{l, m \pm 1, n-1}\right), \\
& \bar{U}_{z l, m, n \pm 1}=\frac{1}{2 h}\left( \pm 3 U_{l, m, n \pm 1} \mp 4 U_{l, m, n} \pm U_{l, m, n \mp 1}\right) \text {, } \\
& \bar{V}_{z l, m, n \pm 1}=\frac{1}{2 h}\left( \pm 3 V_{l, m, n \pm 1} \mp 4 V_{l, m, n} \pm V_{l, m, n \mp 1}\right), \\
& \bar{U}_{x x l, m \pm 1, n}=\frac{1}{h^{2}}\left(U_{l+1, m \pm 1, n}-2 U_{l, m \pm 1, n}+U_{l-1, m \pm 1, n}\right) \text {, } \\
& \bar{V}_{x x l, m \pm 1, n}=\frac{1}{h^{2}}\left(V_{l+1, m \pm 1, n}-2 V_{l, m \pm 1, n}+V_{l-1, m \pm 1, n}\right) \text {, } \\
& \bar{U}_{x x l, m, n \pm 1}=\frac{1}{h^{2}}\left(U_{l+1, m, n \pm 1}-2 U_{l, m, n \pm 1}+U_{l-1, m, n \pm 1}\right) \text {, } \\
& \bar{V}_{x x l, m, n \pm 1}=\frac{1}{h^{2}}\left(V_{l+1, m, n \pm 1}-2 V_{l, m, n \pm 1}+V_{l-1, m, n \pm 1}\right), \\
& \bar{U}_{y y l \pm 1, m, n}=\frac{1}{h^{2}}\left(U_{l \pm 1, m+1, n}-2 U_{l \pm 1, m, n}+U_{l \pm 1, m-1, n}\right) \text {, } \\
& \bar{V}_{y y l \pm 1, m, n}=\frac{1}{h^{2}}\left(V_{l \pm 1, m+1, n}-2 V_{l \pm 1, m, n}+V_{l \pm 1, m-1, n}\right), \\
& \bar{U}_{y y l, m, n \pm 1}=\frac{1}{h^{2}}\left(U_{l, m+1, n \pm 1}-2 U_{l, m, n \pm 1}+U_{l, m-1, n \pm 1}\right) \text {, } \\
& \bar{V}_{y y l, m, n \pm 1}=\frac{1}{h^{2}}\left(V_{l, m+1, n \pm 1}-2 V_{l, m, n \pm 1}+V_{l, m-1, n \pm 1}\right) \text {, } \\
& \bar{U}_{z z l \pm 1, m, n}=\frac{1}{h^{2}}\left(U_{l \pm 1, m, n+1}-2 U_{l \pm 1, m, n}+U_{l \pm 1, m, n-1}\right), \\
& \bar{V}_{z z l \pm 1, m, n}=\frac{1}{h^{2}}\left(V_{l \pm 1, m, n+1}-2 V_{l \pm 1, m, n}+V_{l \pm 1, m, n-1}\right) \text {, } \\
& \bar{U}_{z z l, m \pm 1, n}=\frac{1}{h^{2}}\left(U_{l, m \pm 1, n+1}-2 U_{l, m \pm 1, n}+U_{l, m \pm 1, n-1}\right), \\
& \bar{V}_{z z l, m \pm 1, n}=\frac{1}{h^{2}}\left(V_{l, m \pm 1, n+1}-2 V_{l, m \pm 1, n}+V_{l, m \pm 1, n-1}\right) \text {. }
\end{aligned}
$$$$
\bar{F}_{l \pm 1, m, n}=f\left(x_{l \pm 1}, y_{m}, z_{n}, U_{l \pm 1, m, n}, V_{l \pm 1, m, n}, \bar{U}_{x l \pm 1, m, n}, \bar{V}_{x l \pm 1, m, n}, \bar{U}_{y l \pm 1, m, n}, \bar{V}_{y l \pm 1, m, n}, \bar{U}_{z l \pm 1, m, n}, \bar{V}_{z l \pm 1, m, n}\right)
$$$$
\bar{F}_{l, m \pm 1, n}=f\left(x_{l}, y_{m \pm 1}, z_{n}, U_{l, m \pm 1, n}, V_{l, m \pm 1, n}, \bar{U}_{x l, m \pm 1, n}, \bar{V}_{x l, m \pm 1, n}, \bar{U}_{y l, m \pm 1, n}, \bar{V}_{y l, m \pm 1, n}, \bar{U}_{z l, m \pm 1, n}, \bar{V}_{z l, m \pm 1, n}\right)
$$$$
\bar{F}_{l, m, n \pm 1}=f\left(x_{l}, y_{m}, z_{n \pm 1}, U_{l, m, n \pm 1}, V_{l, m, n \pm 1}, \bar{U}_{x l, m, n \pm 1}, \bar{V}_{x l, m, n \pm 1}, \bar{U}_{y l, m, n \pm 1}, \bar{V}_{y l, m, n \pm 1}, \bar{U}_{z l, m, n \pm 1}, \bar{V}_{z l, m, n \pm 1}\right)
$$

Then we need to evaluate 


$$
\begin{aligned}
& \overline{\bar{U}}_{y l, m, n}=\bar{U}_{y l, m, n}-\frac{h}{12}\left(V_{l, m+1, n}-V_{l, m-1, n}\right)+\frac{h}{12}\left(\bar{U}_{x x l, m+1, n}-\bar{U}_{x x l, m-1, n}\right)+\frac{h}{12}\left(\bar{U}_{z z l, m+1, n}-\bar{U}_{z z l, m-1, n}\right) \\
& \overline{\bar{V}}_{y l, m, n}=\bar{V}_{y l, m, n}-\frac{h}{12}\left(\bar{F}_{l, m+1, n}-\bar{F}_{l, m-1, n}\right)+\frac{h}{12}\left(\bar{V}_{x x l, m+1, n}-\bar{V}_{x x l, m-1, n}\right)+\frac{h}{12}\left(\bar{V}_{z z l, m+1, n}-\bar{V}_{z z l, m-1, n}\right) \\
& \overline{\bar{U}}_{z l, m, n}=\bar{U}_{z l, m, n}-\frac{h}{12}\left(V_{l, m, n+1}-V_{l, m, n-1}\right)+\frac{h}{12}\left(\bar{U}_{x x l, m, n+1}-\bar{U}_{x x l, m, n-1}\right)+\frac{h}{12}\left(\bar{U}_{y y l, m, n+1}-\bar{U}_{y y l, m, n-1}\right) \\
& \overline{\bar{V}}_{z l, m, n}=\bar{V}_{z l, m, n}-\frac{h}{12}\left(\bar{F}_{l, m, n+1}-\bar{F}_{l, m, n-1}\right)+\frac{h}{12}\left(\bar{V}_{x x l, m, n+1}-\bar{V}_{x x l, m, n-1}\right)+\frac{h}{12}\left(\bar{V}_{y y l, m, n+1}-\bar{V}_{y y l, m, n-1}\right)
\end{aligned}
$$

Finally, let

$$
\overline{\bar{F}}_{l, m, n}=f\left(x_{l}, y_{m}, z_{n}, U_{l, m, n}, V_{l, m, n}, \overline{\bar{U}}_{x l, m, n}, \overline{\bar{V}}_{x l, m, n}, \overline{\bar{U}}_{y l, m, n}, \overline{\bar{V}}_{y l, m, n}, \overline{\bar{U}}_{z l, m, n}, \overline{\bar{V}}_{z l, m, n}\right)
$$

Then at each internal grid point $\left(x_{l}, y_{m}, z_{n}\right)$ of the solu- tions (3) are discretized by tion region $\Omega$, the given system of differential Equa-

$$
\begin{aligned}
L[U] \equiv & U_{l, m-1, n-1}+U_{l-1, m, n-1}+2 U_{l, m, n-1}+U_{l+1, m, n-1}+U_{l, m+1, n-1}+U_{l-1, m-1, n}+2 U_{l, m-1, n}+U_{l+1, m-1, n}+2 U_{l-1, m, n} \\
& -24 U_{l, m, n}+2 U_{l+1, m, n}+U_{l-1, m+1, n}+2 U_{l, m+1, n}+U_{l+1, m+1, n}+U_{l, m-1, n+1}+U_{l-1, m, n+1}+2 U_{l, m, n+1}+U_{l+1, m, n+1}+U_{l, m+1, n+1} \\
& =\frac{h^{2}}{2}\left[V_{l+1, m, n}+V_{l-1, m, n}+V_{l, m+1, n}+V_{l, m-1, n}+V_{l, m, n+1}+V_{l, m, n-1}+6 V_{l, m, n}\right]+O\left(h^{6}\right) \\
l, m, n= & 1(1) N \\
L[V] \equiv & V_{l, m-1, n-1}+V_{l-1, m, n-1}+2 V_{l, m, n-1}+V_{l+1, m, n-1}+V_{l, m+1, n-1}+V_{l-1, m-1, n}+2 V_{l, m-1, n}+V_{l+1, m-1, n}+2 V_{l-1, m, n}-24 V_{l, m, n} \\
& +2 V_{l+1, m, n}+V_{l-1, m+1, n}+2 V_{l, m+1, n}+V_{l+1, m+1, n}+V_{l, m-1, n+1}+V_{l-1, m, n+1}+2 V_{l, m, n+1}+V_{l+1, m, n+1}+V_{l, m+1, n+1} \\
= & \frac{h^{2}}{2}\left[\bar{F}_{l+1, m, n}+\bar{F}_{l-1, m, n}+\bar{F}_{l, m+1, n}+\bar{F}_{l, m-1, n}+\bar{F}_{l, m, n+1}+\bar{F}_{l, m, n-1}+6 \bar{F}_{l, m, n}\right]+\bar{T}_{l, m, n}, \\
l, m, n= & 1(1) N
\end{aligned}
$$

where $\bar{T}_{l, m, n}=O\left(h^{6}\right)$. Note that, the approximations (30a) and (30b) require only 19-grid points with a single computational cell. Incorporating the Dirichlet boundary conditions given by (4) into the difference methods (30a) and (30b), we obtain the sparse system of tri-block-block diagonal matrix equations in coupled form, which can be solved by appropriate iterative methods (see [20-24]).

\section{Derivation of the Fourth Order Approximations}

At the grid point $\left(x_{l}, y_{m}, z_{n}\right)$, let us denote

$$
\begin{aligned}
& U_{i j k}=\frac{\partial^{i+j+k} U}{\partial x_{l}^{i} \partial y_{m}^{j} \partial z_{n}{ }^{j}}, \alpha_{l, m, n}^{(1)}=\frac{\partial f}{\partial U_{x l, m, n}}, \alpha_{l, m, n}^{(2)}=\frac{\partial f}{\partial V_{x l, m, n}}, \beta_{l, m, n}^{(1)}=\frac{\partial f}{\partial U_{y l, m, n}}, \beta_{l, m, n}^{(2)}=\frac{\partial f}{\partial V_{y l, m, n}}, \\
& \gamma_{l, m, n}^{(1)}=\frac{\partial f}{\partial U_{z l, m, n}}, \gamma_{l, m, n}^{(2)}=\frac{\partial f}{\partial V_{z l, m, n}}
\end{aligned}
$$

Further, at the grid point $\left(x_{l}, y_{m}, z_{n}\right)$, we define

$$
F_{l, m, n}=f\left(x_{l}, y_{m}, z_{n}, U_{l, m, n}, V_{l, m, n}, U_{x l, m, n}, V_{x l, m, n}, U_{y l, m, n}, V_{y l, m, n}, U_{z l, m, n}, V_{z l, m, n}\right)
$$

Using Taylor expansion about the grid point $\left(x_{l}, y_{m}, z_{n}\right)$, we first obtain

$$
L[V]=\frac{h^{2}}{2}\left[F_{l+1, m, n}+F_{l-1, m, n}+F_{l, m+1, n}+F_{l, m-1, n}+F_{l, m, n+1}+F_{l, m, n-1}+6 F_{l, m, n}\right]+O\left(h^{6}\right)
$$


Now by the help of the approximations (8a)-(16b), from (23)-(25), we obtain

$$
\begin{aligned}
& \bar{F}_{l \pm 1, m, n}=F_{l \pm 1, m, n}+\frac{h^{2}}{6} T_{1} \pm O\left(h^{3}\right), \\
& \bar{F}_{l, m \pm 1, n}=F_{l, m \pm 1, n}+\frac{h^{2}}{6} T_{2} \pm O\left(h^{3}\right), \\
& \bar{F}_{l, m, n \pm 1}=F_{l, m, n \pm 1}+\frac{h^{2}}{6} T_{3} \pm O\left(h^{3}\right) .
\end{aligned}
$$

where

$$
\begin{aligned}
T_{1}= & -2 U_{300} \alpha_{l, m, n}^{(1)}-2 V_{300} \alpha_{l, m, n}^{(2)}+U_{030} \beta_{l, m, n}^{(1)}, \\
+ & V_{030} \beta_{l, m, n}^{(2)}+U_{003} \gamma_{l, m, n}^{(1)}+V_{003} \gamma_{l, m, n}^{(2)} \\
T_{2}= & U_{300} \alpha_{l, m, n}^{(1)}+V_{300} \alpha_{l, m, n}^{(2)}-2 U_{030} \beta_{l, m, n}^{(1)}, \\
& -2 V_{030} \beta_{l, m, n}^{(2)}+U_{003} \gamma_{l, m, n}^{(1)}+V_{003} \gamma_{l, m, n}^{(2)} \\
T_{3}= & U_{300} \alpha_{l, m, n}^{(1)}+V_{300} \alpha_{l, m, n}^{(2)}+U_{030} \beta_{l, m, n}^{(1)} \\
& +V_{030} \beta_{l, m, n}^{(2)}-2 U_{003} \gamma_{l, m, n}^{(1)}-2 V_{003} \gamma_{l, m, n}^{(2)}
\end{aligned}
$$

Let us consider the linear combination

$$
\begin{aligned}
& \overline{\bar{U}}_{x l, m, n}=\bar{U}_{x l, m, n}+h a_{11}\left(V_{l+1, m, n}-V_{l-1, m, n}\right) \\
& +h a_{12}\left(\bar{U}_{y y l+1, m, n}-\bar{U}_{y y l-1, m, n}\right) \\
& +h a_{13}\left(\bar{U}_{z z l+1, m, n}-\bar{U}_{z z l-1, m, n}\right) \\
& \overline{\bar{V}}_{x l, m, n}=\bar{V}_{x l, m, n}+h b_{11}\left(\bar{F}_{l+1, m, n}-\bar{F}_{l-1, m, n}\right) \\
& +h b_{12}\left(\bar{V}_{y y l+1, m, n}-\bar{V}_{y y l-1, m, n}\right) \quad, \\
& +h b_{13}\left(\bar{V}_{\mathrm{zzl} l+1, m, n}-\bar{V}_{\mathrm{zzl} l-1, m, n}\right) \\
& \overline{\bar{U}}_{y l, m, n}=\bar{U}_{y l, m, n}+h a_{21}\left(V_{l, m+1, n}-V_{l, m-1, n}\right) \\
& +h a_{22}\left(\bar{U}_{x x l, m+1, n}-\bar{U}_{x x l, m-1, n}\right), \\
& +h a_{23}\left(\bar{U}_{z z l, m+1, n}-\bar{U}_{z z l, m-1, n}\right) \\
& \overline{\bar{V}}_{y l, m, n}=\bar{V}_{y l, m, n}+h b_{21}\left(\bar{F}_{l, m+1, n}-\bar{F}_{l, m-1, n}\right) \\
& +h b_{22}\left(\bar{V}_{x x l, m+1, n}-\bar{V}_{x x l, m-1, n}\right) \quad, \\
& +h b_{23}\left(\bar{V}_{z z l, m+1, n}-\bar{V}_{z z l, m-1, n}\right) \\
& \overline{\bar{U}}_{z l, m, n}=\bar{U}_{z l, m, n}+h a_{31}\left(V_{l, m, n+1}-V_{l, m, n-1}\right) \\
& +h a_{32}\left(\bar{U}_{x x l, m, n+1}-\bar{U}_{x x l, m, n-1}\right), \\
& +h a_{33}\left(\bar{U}_{y y l, m, n+1}-\bar{U}_{y y l, m, n-1}\right) \\
& \overline{\bar{V}}_{z l, m, n}=\bar{V}_{z l, m, n}+h b_{31}\left(\bar{F}_{l, m, n+1}-\bar{F}_{l, m, n-1}\right) \\
& +h b_{32}\left(\bar{V}_{x x l, m, n+1}-\bar{V}_{x x l, m, n-1}\right) \\
& +h b_{33}\left(\bar{V}_{y y l, m, n+1}-\bar{V}_{y y l, m, n-1}\right)
\end{aligned}
$$

where $a_{i j}$ and $b_{i j} ; i, j=1,2,3$ are parameters to be determined.

Now using the approximations (5a)-(7b), (17a)-(22b) and (34)-(36), from (37a)-(39b), we obtain

$$
\begin{aligned}
& \overline{\bar{U}}_{x l, m, n}=U_{x l, m, n}+\frac{h^{2}}{6} T_{4}+O\left(h^{4}\right), \\
& \overline{\bar{V}}_{x l, m, n}=V_{x l, m, n}+\frac{h^{2}}{6} T_{5}+O\left(h^{4}\right), \\
& \overline{\bar{U}}_{y l, m, n}=U_{y l, m, n}+\frac{h^{2}}{6} T_{6}+O\left(h^{4}\right), \\
& \overline{\bar{V}}_{y l, m, n}=V_{y l, m, n}+\frac{h^{2}}{6} T_{7}+O\left(h^{4}\right), \\
& \overline{\bar{U}}_{z l, m, n}=U_{z l, m, n}+\frac{h^{2}}{6} T_{8}+O\left(h^{4}\right), \\
& \overline{\bar{V}}_{z l, m, n}=V_{z l, m, n}+\frac{h^{2}}{6} T_{9}+O\left(h^{4}\right) .
\end{aligned}
$$

where

$$
\begin{gathered}
T_{4}=\left(1+12 a_{11}\right) U_{300}+12\left(a_{11}+a_{12}\right) U_{120}, \\
+12\left(a_{11}+a_{13}\right) U_{102} \\
T_{5}=\left(1+12 b_{11}\right) V_{300}+12\left(b_{11}+b_{12}\right) V_{120}+12\left(b_{11}+b_{13}\right) V_{102}, \\
T_{6}=\left(1+12 a_{21}\right) U_{030}+12\left(a_{21}+a_{22}\right) U_{210}, \\
+12\left(a_{21}+a_{23}\right) U_{012} \\
T_{7}=\left(1+12 b_{21}\right) V_{030}+12\left(b_{21}+b_{22}\right) V_{210}+12\left(b_{21}+b_{23}\right) V_{012}, \\
T_{8}=\left(1+12 a_{31}\right) U_{003}+12\left(a_{31}+a_{32}\right) U_{201}, \\
+12\left(a_{31}+a_{33}\right) U_{021} \\
T_{9}=\left(1+12 b_{31}\right) V_{003}+12\left(b_{31}+b_{32}\right) V_{201}+12\left(b_{31}+b_{33}\right) V_{021} .
\end{gathered}
$$

By the help of the approximations (40a)-(42b), from (29), we get

$$
\overline{\bar{F}}_{l, m, n}=F_{l, m, n}+\frac{h^{2}}{6} T_{10}+O\left(h^{4}\right)
$$

where

$$
\begin{aligned}
T_{10}= & T_{4} \alpha_{l, m, n}^{(1)}+T_{5} \alpha_{l, m, n}^{(2)}+T_{6} \beta_{l, m, n}^{(1)}+T_{7} \beta_{l, m, n}^{(2)} \\
& +T_{8} \gamma_{l, m, n}^{(1)}+T_{9} \gamma_{l, m, n}^{(2)}
\end{aligned}
$$

Finally, by the help of the relations (34)-(36) and (43), from (30b) and (33), we obtain the local truncation error

$$
\bar{T}_{l, m, n}=\frac{-h^{4}}{6}\left[T_{1}+T_{2}+T_{3}+3 T_{10}\right]+O\left(h^{6}\right)
$$

The proposed difference methods (30a) and (30b) are to be of $O\left(h^{4}\right)$, the coefficient of $h^{4}$ in (44) must be zero and we obtain a relation 


$$
T_{1}+T_{2}+T_{3}+3 T_{10}=0
$$

Substituting the values of $T_{1}, T_{2}, T_{3}$ and $T_{10}$ in (45), we obtain the values of parameters $a_{i 1}=b_{i 1}=\frac{-1}{12}$ for $i=$ $1,2,3$ and $a_{i j}=b_{i j}=\frac{1}{12}$ for $i=1,2,3$ and $j=2,3$, and the local truncation error (44) reduces to $\bar{T}_{l, m, n}=O\left(h^{6}\right)$.

We may summarize the results as follows:

Theorem 1: The compact 19-point finite difference methods (30a) and (30b) with the approximations listed in (5a)-(29) are of $O\left(h^{4}\right)$ for the numerical solutions of $u(x, y, z)$ and its Laplacian $\nabla^{2} u(x, y, z)$ of the non-linear biharmonic Equation (1) with Dirichlet boundary conditions (2).

\section{Stability Consideration and Results of Computational Experiments}

Let us consider the test equation

$$
\nabla^{4} u(x, y, z)=G(x, y, z), 0<x, y, z<1
$$

Applying the proposed method (30a) and (30b) to the above equation, we obtain

$$
\begin{aligned}
& U_{l, m-1, n-1}+U_{l-1, m, n-1}+2 U_{l, m, n-1}+U_{l+1, m, n-1} \\
& +U_{l, m+1, n-1}+U_{l-1, m-1, n}+2 U_{l, m-1, n}+U_{l+1, m-1, n} \\
& +2 U_{l-1, m, n}-24 U_{l, m, n}+2 U_{l+1, m, n}+U_{l-1, m+1, n} \\
& +2 U_{l, m+1, n}+U_{l+1, m+1, n}+U_{l, m-1, n+1}+U_{l-1, m, n+1} \\
& +2 U_{l, m, n+1}+U_{l+1, m, n+1}+U_{l, m+1, n+1} \\
& =\frac{h^{2}}{2}\left[V_{l+1, m, n}+V_{l-1, m, n}+V_{l, m+1, n}+V_{l, m-1, n}\right. \\
& \left.+V_{l, m, n+1}+V_{l, m, n-1}+6 V_{l, m, n}\right] \\
& l, m, n=1(1) N \\
& V_{l, m-1, n-1}+V_{l-1, m, n-1}+2 V_{l, m, n-1}+V_{l+1, m, n-1} \\
& +V_{l, m+1, n-1}+V_{l-1, m-1, n}+2 V_{l, m-1, n}+V_{l+1, m-1, n} \\
& +2 V_{l-1, m, n}-24 V_{l, m, n}+2 V_{l+1, m, n}+V_{l-1, m+1, n} \\
& +2 V_{l, m+1, n}+V_{l+1, m+1, n}+V_{l, m-1, n+1}+V_{l-1, m, n+1} \\
& +2 V_{l, m, n+1}+V_{l+1, m, n+1}+V_{l, m+1, n+1} \\
& =\frac{h^{2}}{2}\left[G_{l+1, m, n}+G_{l-1, m, n}+G_{l, m+1, n}+G_{l, m-1, n}\right. \\
& \left.+G_{l, m, n+1}+G_{l, m, n-1}+6 G_{l, m, n}\right] \\
& \\
& l, m, n=1(1) N
\end{aligned}
$$

where $G_{l, m, n}=G\left(x_{l}, y_{m}, z_{n}\right), \ldots$ etc.

An iterative method for (47a) and (47b) can be written as

$$
24 \boldsymbol{I} \boldsymbol{u}^{(\mathrm{k}+1)}=\boldsymbol{A} \boldsymbol{u}^{(\mathrm{k})}-\frac{h^{2}}{2} \boldsymbol{B} \boldsymbol{v}^{(\mathrm{k})}+\boldsymbol{R} \boldsymbol{H} \boldsymbol{U}
$$

$$
24 \boldsymbol{I} \boldsymbol{v}^{(\mathrm{k}+1)}=\boldsymbol{O} \boldsymbol{u}^{(\mathrm{k})}+\boldsymbol{A} \boldsymbol{v}^{(\mathrm{k})}+\boldsymbol{R} \boldsymbol{H} \boldsymbol{V}
$$

where $\boldsymbol{u}^{(k)}, \boldsymbol{v}^{(k)}$ are solution vectors and $\boldsymbol{R} \boldsymbol{H} \boldsymbol{U}, \boldsymbol{R} \boldsymbol{H} \boldsymbol{V}$ are right hand side vectors consists of boundary and homogenous function values.

Above iterative method in matrix form can be written as

$$
\left[\begin{array}{l}
\boldsymbol{U}^{(\mathrm{k}+1)} \\
\boldsymbol{V}^{(\mathrm{k}+1)}
\end{array}\right]=\boldsymbol{G}\left[\begin{array}{l}
\boldsymbol{U}^{(\mathrm{k})} \\
\boldsymbol{V}^{(\mathrm{k})}
\end{array}\right]+\boldsymbol{R} \boldsymbol{H}
$$

where

$$
\boldsymbol{G}=\frac{1}{24}\left[\begin{array}{cc}
\boldsymbol{A} & \frac{-h^{2}}{2} \boldsymbol{B} \\
\boldsymbol{0} & \boldsymbol{A}
\end{array}\right], R H=\left[\begin{array}{l}
\boldsymbol{R} \boldsymbol{H} \boldsymbol{U} \\
\boldsymbol{R H V}
\end{array}\right] .
$$

We denote $\boldsymbol{I}=[0,1,0]$ as the $N$ th order identity matrix and $\boldsymbol{H}=[1,2,1]$ and $\boldsymbol{Q}=[2,0,2]$ as $N$ th order tridiagonal matrices, and $\boldsymbol{C}=[\boldsymbol{I}, \boldsymbol{H}, \boldsymbol{I}]$ and $\boldsymbol{D}=[\boldsymbol{H}, \boldsymbol{Q}, \boldsymbol{H}]$ as the Nth order block-tridiagonal matrices, where, in general, we denote

$$
[a, b, c]=\left[\begin{array}{ccccc}
b & c & & & \mathbf{0} \\
a & b & c & & \\
& & \ddots & & \\
& & a & b & c \\
\mathbf{0} & & & a & b
\end{array}\right]_{N \times N}
$$

as $N$ th order tridiagonal matrix whose eigen values are given by

$$
b+2 \sqrt{a c} \cos \left(\frac{\pi j}{N+1}\right), j=1,2, \cdots N .
$$

The eigenvalues of $\boldsymbol{I}, \boldsymbol{H}$ and $\boldsymbol{Q}$ are $1(\mathrm{~N}$-times), $2+2 \cos (k \pi h)$ and $4 \cos (k \pi h), k=1(1) N$ respectively, where $(N+1) h=1$.

The eigenvalues of $\boldsymbol{C}$ and $\boldsymbol{D}$ are given by

$$
\xi_{j k}=2+2[\cos (j \pi h)+\cos (k \pi h)] ; j, k=1(1) N
$$

and

$$
\begin{aligned}
& \left.\eta_{j k}=4[\cos (j \pi h)+\cos (k \pi h))+\cos (j \pi h) \cos (k \pi h)\right] \\
& j, k=1(1) N
\end{aligned}
$$

The matrix $\boldsymbol{A}=[\boldsymbol{C}, \boldsymbol{D}, \boldsymbol{C}]$ associated with the iteration matrix $\boldsymbol{G}$ is a $N$ th order block-block-tridiagonal matrix whose eigenvalues are given by

$$
\gamma_{i j k}=\eta_{j k}+2 \xi_{j k} \cos (i \pi h) ; i, j, k=1(1) N
$$

or,

$$
\begin{aligned}
\gamma_{i j k}= & 4[\cos (i \pi h)+\cos (j \pi h) \\
& +\cos (k \pi h)+\cos (i \pi h) \cos (j \pi h) \\
& +\cos (j \pi h) \cos (k \pi h)+\cos (k \pi h) \cos (i \pi h)] ; \\
i, j, k= & 1(1) N
\end{aligned}
$$


Similarly, the eigenvalues of the matrix $\boldsymbol{B}$ associated with the iteration matrix $\boldsymbol{G}$ are given by

$$
\begin{aligned}
& \sigma_{i j k}=6+2[\cos (i \pi h)+\cos (j \pi h)+\cos (k \pi h)] ; \\
& i, j, k=1(1) N
\end{aligned}
$$

The iterative method (49) is stable as long as $\rho(\boldsymbol{G}) \leq 1$, where $\rho(\boldsymbol{G})$ is the spectral radius of $\boldsymbol{G}$.

The characteristic equation of the matrix $\boldsymbol{G}$ is given by

$$
\operatorname{det}\left[\begin{array}{cc}
\frac{1}{24} \gamma_{i j k}-\lambda & \frac{-h^{2}}{48} \sigma_{i j k} \\
\mathbf{0} & \frac{1}{24} \gamma_{i j k}-\lambda
\end{array}\right]=0 ; i, j, k=1(1) N
$$

Thus the eigenvalues of $\boldsymbol{G}$ are given by

$\lambda_{i j k}=\lambda=\frac{1}{24} \gamma_{i j k}=\frac{1}{6}[\cos (i \pi h)+\cos (j \pi h)+\cos (k \pi h)+\cos (i \pi h) \cos (j \pi h)+\cos (j \pi h) \cos (k \pi h)+\cos (k \pi h) \cos (i \pi h)]$

The maximum values of all eigenvalues of $\boldsymbol{G}$ occur at $i=j=k=1$.

Hence,

$$
\begin{aligned}
\rho(\boldsymbol{G})=\max .\left|\lambda_{i j k}\right| & =\frac{\cos (\pi h)}{2}[1+\cos (\pi h)] \leq 1, \quad(56) \quad \begin{array}{l}
\text { differential Equations (3a) and (3b) are } \\
\text { and can be written in a coupled manner }
\end{array} \\
& U_{l, m, n-1}+U_{l, m-1, n}+U_{l-1, m, n}-6 U_{l, m, n}+U_{l+1, m, n}+U_{l, m+1, n}+U_{l, m, n+1}=h^{2} V_{l, m, n}+O\left(h^{4}\right), \\
& l, m, n=1(1) N \\
& V_{l, m, n-1}+V_{l, m-1, n}+V_{l-1, m, n}-6 V_{l, m, n}+V_{l+1, m, n}+V_{l, m+1, n}+V_{l, m, n+1} \\
& =h^{2} f\left(x_{l}, y_{m}, z_{n}, U_{l, m, n}, V_{l, m, n}, \bar{U}_{x l, m, n}, \bar{V}_{x l, m, n}, \bar{U}_{y l, m, n}, \bar{V}_{l l, m, n}, \bar{U}_{z l, m, n}, \bar{V}_{l l, m, n}\right)+O\left(h^{4}\right) \\
& l, m, n=1(1) N
\end{aligned}
$$
iterative method (48a)-(48b) is stable.

The second order approximations for the system of differential Equations (3a) and (3b) are straightforward

Note that, the second order approximations (57a) and (57b) require only 7 -grid points on a single computational cell (see Figure 1).

By combining the difference equations at each internal grid points, we obtain a large sparse system of matrix to solve. At each interior mesh point, we have two unknowns $u$ and $\nabla^{2} u \equiv v$, that is, the number of bands with non-zero entries is increased, and so is the size of the final matrix for the same mesh size. However, by this new method, the value of the Laplacian, which is often of interest, is also computed.

Whenever $f\left(x, y, z, u, v, u_{x}, v_{x}, u_{y}, v_{y}, u_{z}, v_{z}\right)$ is linear (or, non-linear) in $u, v, u_{x}, v_{x}, u_{y}, v_{y}, u_{z}$ and $v_{z}$, the difference Equations (30a) and (30b) form a linear (or, non-linear) system. To solve such a system or indeed to demonstrate the existence of a solution, we use iterative methods. In this section, we solve the following three test problems in the region $0<x, y, z<1$, whose exact solutions are known. The Dirichlet boundary conditions and right hand side homogeneous functions are obtained by using the exact solutions. We have also compared the numerical results obtained by proposed fourth order approximations (30a) and (30b) with the numerical results obtained by corresponding second order approximations (57a) and (57b). In all cases, we have considered $\boldsymbol{u}^{(0)}=\mathbf{0}$ as the initial approximation and the iterations were stopped when the absolute error tolerance $\left|\boldsymbol{u}^{(k+1)}-\boldsymbol{u}^{(k)}\right| \leq 10^{-12}$ was achieved. In all cases, we have calculated maximum absolute errors $\left(l_{\infty}\right.$-norm) for different grid sizes. All computations were performed using

\begin{tabular}{|c|c|c|c|}
\hline \multicolumn{2}{|c|}{$h$} & $O\left(h^{4}\right)$-method & $O\left(h^{2}\right)$-method \\
\hline $1 / 8$ & $\begin{array}{c}U \\
\nabla^{2} u\end{array}$ & $\begin{array}{l}0.1065(-01) \\
0.6145(+00)\end{array}$ & $\begin{array}{l}0.1089(-01) \\
0.2932(+02)\end{array}$ \\
\hline $1 / 16$ & $\begin{array}{c}U \\
\nabla^{2} u\end{array}$ & $\begin{array}{l}0.6659(-03) \\
0.3776(-01)\end{array}$ & $\begin{array}{l}0.2614(+00) \\
0.7121(+01)\end{array}$ \\
\hline $1 / 32$ & $\begin{array}{c}U \\
\nabla^{2} u\end{array}$ & $\begin{array}{l}0.4157(-04) \\
0.2349(\mathrm{v} 02)\end{array}$ & $\begin{array}{l}0.6469(-01) \\
0.1767(+01)\end{array}$ \\
\hline $1 / 64$ & $\begin{array}{c}U \\
\nabla^{2} u\end{array}$ & $\begin{array}{l}0.2586(-05) \\
0.1466(-03)\end{array}$ & $\begin{array}{l}0.1611(-01) \\
0.4410(+00)\end{array}$ \\
\hline
\end{tabular}
double precision arithmetic.

Test Problem 1: (Biharmonic problem)

$$
\begin{aligned}
& \frac{\partial^{4} u}{\partial x^{4}}+\frac{\partial^{4} u}{\partial y^{4}}+\frac{\partial^{4} u}{\partial z^{4}}+2\left(\frac{\partial^{4} u}{\partial x^{2} \partial y^{2}}+\frac{\partial^{4} u}{\partial y^{2} \partial z^{2}}+\frac{\partial^{4} u}{\partial z^{2} \partial x^{2}}\right) \\
& =G(x, y, z), 0<x, y, z<1
\end{aligned}
$$

The exact solution is

$$
u(x, y, z)=(1-\cos 2 \pi x)(1-\cos 2 \pi y)(1-\cos 2 \pi z) .
$$

The maximum absolute errors are tabulated in Table 1.

Table 1. Test Problem 1: The maximum absolute errors. 
Test Problem 2: (Variable coefficient problems)

$$
\begin{aligned}
\nabla^{4} u & =\left(1+x^{2}\right)\left(u_{x x x}+u_{x y y}+u_{x z z}\right) \\
& +\left(1+y^{2}\right)\left(u_{x x y}+u_{y y y}+u_{y z z}\right) \\
& +\left(1+z^{2}\right)\left(u_{x x z}+u_{y y z}+u_{z z z}\right) \\
& +\left(1+\sin ^{2} x\right) u_{x}+\left(1+\sin ^{2} y\right) u_{y} \\
& +\left(1+\sin ^{2} z\right) u_{z}+G(x, y, z), \\
0 & <x, y, z<1
\end{aligned}
$$

The exact solution is

$$
\begin{aligned}
u( & x, y, z)=\sin (\pi x) \cdot \sin (\pi y) \cdot \sin (\pi z) . \\
\nabla^{4} u= & \left(1+\cos ^{2} x\right)\left(u_{x x x}+u_{x y y}+u_{x z z}\right) \\
& +\left(1+\cos ^{2} y\right)\left(u_{x x y}+u_{y y y}+u_{y z z}\right) \\
& +\left(1+\cos ^{2} z\right)\left(u_{x x z}+u_{y y z}+u_{z z z}\right) \\
& +\left(1+x^{4}\right) u_{x}+\left(1+y^{4}\right) u_{y}+\left(1+z^{4}\right) u_{z} \\
& +G(x, y, z), 0<x, y, z<1
\end{aligned}
$$

The exact solution is $u(x, y, z)=\mathrm{e}^{x+y+z}$.

The maximum absolute errors are tabulated in Table 2.
Test Problem 3: (Navier-Stokes model equation in terms of stream function $\psi$ ) (see [25])

$$
\begin{gathered}
\frac{1}{R_{e}} \nabla^{4} \psi=\psi_{y}\left(\psi_{x x x}+\psi_{x y y}\right)-\psi_{x}\left(\psi_{x x y}+\psi_{y y y}\right) \\
+G(x, y, z), 0<x, y, z<1
\end{gathered}
$$

The exact solution is $\psi(x, y, z)=\mathrm{e}^{x} \sin (\pi y) \sin (\pi z)$.

The maximum absolute errors are tabulated in Table 3 for various values of Reynolds number $R_{e}$.

\section{Concluding Remarks}

\begin{tabular}{|c|c|c|c|c|c|}
\hline \multicolumn{2}{|c|}{$h$} & \multicolumn{2}{|c|}{ Problem (59a) } & \multicolumn{2}{|c|}{ Problem (59b) } \\
\hline & & $O\left(h^{4}\right)$-Method & $O\left(h^{2}\right)$-Method & $O\left(h^{4}\right)$-Method & $O\left(h^{2}\right)$-Method \\
\hline \multirow{2}{*}{$1 / 4$} & $u$ & $0.7778(-02)$ & $0.1078(+00)$ & $0.1464(-04)$ & $0.5088(-02)$ \\
\hline & $\nabla^{2} u$ & $0.1161(+00)$ & $0.1562(+01)$ & $0.6745(-03)$ & $0.3249(-01)$ \\
\hline \multirow{2}{*}{$1 / 8$} & $U$ & $0.4655(-03)$ & $0.2578(-01)$ & $0.8861(-06)$ & $0.1444(-02)$ \\
\hline & $\nabla^{2} u$ & $0.6952(-02)$ & $0.3812(+00)$ & $0.4195(-04)$ & $0.1016(-01)$ \\
\hline \multirow{2}{*}{$1 / 16$} & $U$ & $0.2877(-04)$ & $0.6377(-02)$ & $0.5529(-07)$ & $0.3699(-03)$ \\
\hline & $\nabla^{2} u$ & $0.4551(-03)$ & $0.9474(-01)$ & $0.2626(-05)$ & $0.2577(-02)$ \\
\hline \multirow{2}{*}{$1 / 32$} & $U$ & $0.1797(-05)$ & $0.1590(-02)$ & $0.3456(-08)$ & $0.9353(-04)$ \\
\hline & $\nabla^{2} u$ & $0.2836(-04)$ & $0.2393(-01)$ & $0.1666(-06)$ & $0.6467(-03)$ \\
\hline
\end{tabular}

In this paper, using coupled approach we discuss a new fourth order 19-point compact finite difference approximation for the solution of 3D non-linear biharmonic elliptic partial differential equations. The method is derived on a single computational cell using the values of $u$ and $\nabla^{2} u$ as the unknowns. We have obtained the numerical solution of $\nabla^{2} u$ as a by-product, which is quite often of interest in many physical problems. Our method is applied to solve several problems including Navier Stokes model equation in terms of stream function $\psi$ and enables us to obtain high accuracy solutions with great efficiency. Numerical results confirm that the pro-

Table 2. Test Problem 2: The maximum absolute errors.

Table 3. Test Problem 3: The maximum absolute errors.

\begin{tabular}{ccccc}
\hline & $h$ & \multicolumn{2}{c}{$O\left(h^{4}\right)$-Method } & $O\left(h^{2}\right)$-Method \\
\cline { 3 - 5 } & & $R_{e}=10^{2}$ & $R_{e}=10^{4}, 10^{6}, 10^{8}$ & $R_{e}=10^{2}, 10^{4}, 10^{6}, 10^{8}$ \\
\hline \multirow{2}{*}{$1 / 8$} & $\psi$ & $0.1808(-03)$ & $0.1880(-03)$ & Over Flow \\
& $\nabla^{2} \psi$ & $0.3332(-02)$ & $0.3524(-02)$ & \\
\multirow{2}{*}{$1 / 16$} & $\psi$ & $0.1134(-04)$ & $0.1202(-04)$ & Over Flow \\
& $\nabla^{2} \psi$ & $0.2079(-03)$ & $0.2253(-03)$ & Over Flow \\
$1 / 32$ & $\psi$ & $0.7095(-06)$ & $0.7545(-06)$ & \\
& $\nabla^{2} \psi$ & $0.1299(-04)$ & $0.1413(-04)$ & Over Flow \\
\hline
\end{tabular}


posed fourth order method produces oscillation free solution for large Reynolds number, whereas the second order method is unstable.

\section{References}

[1] J. Smith, "The Coupled Equation Approach to the Numerical Solution of the Biharmonic Equation by Finite Differences," SIAM Journal on Numerical Analysis, Vol. 7, No. 1, 1970, pp. 104-111. doi:10.1137/0707005

[2] L. W. Ehrlich, "Solving the Biharmonic Equation as Coupled Finite Difference Equations," SIAM Journal on $\mathrm{Nu}$ merical Analysis, Vol. 8, No. 2, 1971, pp. 278-287. doi:10.1137/0708029

[3] L. W. Ehrlich, "Point and Block SOR Applied to a Coupled Set of Difference Equations," Computing, Vol. 12, No. 3, 1974, pp. 181-194. doi:10.1007/BF02293104

[4] L. Bauer and E. L. Riess, "Block Five Diagonal Matrices and the Fast Numerical Solution of the Biharmonic Equation," Mathematics of Computation, Vol. 26, No. 118, 1972, pp. 311-326. doi:10.1090/S0025-5718-1972-0312751-9

[5] R. Glowinski and O. Pironneau, "Numerical Methods for the First Biharmonic Equations and for the Two-Dimensional Stokes Problems," SIAM Review, Vol. 21, No. 2, 1979, pp. 167-212. doi:10.1137/1021028

[6] Y. Kwon, R. Manohar and J. W. Stephenson, "Single Cell Fourth Order Methods for the Biharmonic Equation," Congress Numerantium, Vol. 34, 1982, pp. 475-482.

[7] J. W. Stephenson, "Single Cell Discretization of Order Two and Four for Biharmonic Problems," Journal of Computational Physics, Vol. 55, No. 1, 1984, pp. 65-80. doi:10.1016/0021-9991(84)90015-9

[8] R. K. Mohanty and P. K. Pandey, "Difference Methods of Order Two and Four for Systems of Mildly Non-linear Biharmonic Problems of Second Kind in Two Space Dimensions," Numerical Methods for Partial Differential Equations, Vol. 12, No. 6, 1996, pp. 707-717. doi:10.1002/(SICI)1098-2426(199611)12:6<707::AID-N UM4>3.0.CO;2-W

[9] R. K. Mohanty, M. K. Jain and P. K. Pandey, "Finite Difference Methods of Order Two and Four for 2D Nonlinear Biharmonic Problems of First Kind," International Journal of Computer Mathematics, Vol. 61, No. 1-2, 1996, pp. 155-163. doi:10.1080/00207169608804507

[10] R. K. Mohanty and P. K. Pandey, "Families of Accurate Discretizations of Order Two and Four for 3D Mildly Nonlinear Biharmonic Problems of Second Kind," International Journal of Computer Mathematics, Vol. 68, No. 3-4, 1998, pp. 363-380. doi:10.1080/00207169808804702

[11] D. J. Evans and R. K. Mohanty, "Block Iterative Methods for the Numerical Solution of Two-Dimensional Nonlinear Biharmonic Equations," International Journal of Computer Mathematics, Vol. 69, No. 3-4, 1998, pp. 371-390. doi:10.1080/00207169808804729

[12] R. K. Mohanty, D. J. Evans and P. K. Pandey, "Block Iterative Methods for the Numerical Solution of Three Dimensional Mildly Nonlinear Biharmonic Problems of
First Kind," International Journal of Computer Mathematics, Vol. 77, No. 2, 2001, pp. 319-332. doi: $10.1080 / 00207160108805068$

[13] S. Singh, D. Khattar and R. K. Mohanty, "A New Coupled Approach High Accuracy Numerical Method for the Solution of 2D Nonlinear Biharmonic Equations," Neural Parallel and Scientific Computations, Vol. 17, 2009, pp. 239-256.

[14] D. Khattar, S. Singh and R. K. Mohanty, "A New Coupled Approach High Accuracy Numerical Method for the Solution of 3D Non-Linear Biharmonic Equations," Applied Mathematics and Computations, Vol. 215, No. 8, 2009, pp. 3036-3044. doi:10.1016/j.amc.2009.09.052

[15] R. K. Mohanty, “A New High Accuracy Finite Difference Discretization for the Solution of 2D Non-Linear Biharmonic Equations Using Coupled Approach," Numerical Methods for Partial Differential Equations, Vol. 26, No. 4, 2010, pp. 931-944. doi:10.1002/num.204605

[16] R. K. Mohanty, M. K. Jain and B. N. Mishra, "A Compact Discretization of $O\left(h^{4}\right)$ for Two-Dimensional Nonlinear Triharmonic Equations," Physica Scripta, Vol. 84, No. 2, 2011, pp. 025002.

doi:10.1088/0031-8949/84/02/025002

[17] R. K. Mohanty and S. Dey, "Single Cell Fourth Order Difference Approximations for $(\partial u / \partial x),(\partial u / \partial y)$ and $(\partial u / \partial z)$ of the Three Dimensional Quasi-Linear Elliptic Equation," Numerical Methods for Partial Differential Equations, Vol. 16, No. 5, 2000, pp. 417-425. doi:10.1002/1098-2426(200009)16:5<417::AID-NUM1> 3.0.CO;2-S

[18] R. K. Mohanty, S. Karaa and U. Arora, "Fourth Order Nine Point Unequal Mesh Discretization for the Solution of 2D Non-Linear Elliptic Partial Differential Equations," Neural Parallel and Scientific Computations, Vol. 14, 2006, pp. 453-470.

[19] R. K. Mohanty and S. Singh, "A New Highly Accurate Discretization for Three Dimensional Singularly Perturbed Non-linear Elliptic Partial Differential Equations," Numerical Methods for Partial Differential Equations, Vol. 22, No. 6, 2006, pp. 1379-1395. doi:10.1002/num.20160

[20] L. A. Hageman and D. M. Young, "Applied Iterative Methods," Dover Publications, New York, 2004.

[21] M. K. Jain, "Numerical Solution of Differential Equations," 2nd Edition, John Wiley, New Delhi, 1984.

[22] C. T. Kelly, "Iterative Methods for Linear and Non-Linear Equations," SIAM Publications, Philadelphia, 1995.

[23] Y. Saad, "Iterative Methods for Sparse Linear Systems," SIAM Publications, Philadelphia, 2003. doi: $10.1137 / 1.9780898718003$

[24] G. Meurant, "Computer Solution of Large Linear Systems," North-Holland, Amsterdam, 1999.

[25] W. F. Spotz and G. F. Carey, "High Order Compact Scheme for the Steady Stream-Function Vorticity Equations," International Journal for Numerical Methods in Engineering, Vol. 38, No. 20, 1995, pp. 3497-3512. doi:10.1002/nme.1620382008 